\title{
Hyperpolarizing Gases via Dynamic Nuclear Polarization and Sublimation
}

\author{
A. Comment, ${ }^{1,2,3, *}$ S. Jannin, ${ }^{4}$ J.-N. Hyacinthe,${ }^{5}$ P. Miéville, ${ }^{4}$ R. Sarkar, ${ }^{4}$ P. Ahuja, ${ }^{4}$ P. R. Vasos, ${ }^{4}$ X. Montet, ${ }^{5}$ F. Lazeyras, ${ }^{5}$ \\ J.-P. Vallée, ${ }^{5}$ P. Hautle, ${ }^{6}$ J. A. Konter, ${ }^{6}$ B. van den Brandt, ${ }^{6}$ J.-Ph. Ansermet, ${ }^{3}$ R. Gruetter, ${ }^{1,2,5}$ and G. Bodenhausen ${ }^{4,7, *}$ \\ ${ }^{1}$ Laboratory for Functional and Metabolic Imaging, Ecole Polytechnique Fédérale de Lausanne, CH-1015 Lausanne, Switzerland \\ ${ }^{2}$ Department of Radiology, University of Lausanne, CH-1011 Lausanne, Switzerland \\ ${ }^{3}$ Institute of Condensed Matter Physics, Ecole Polytechnique Fédérale de Lausanne, CH-1015 Lausanne, Switzerland \\ ${ }^{4}$ Institute of Chemical Sciences and Engineering, Ecole Polytechnique Fédérale de Lausanne, CH-1015 Lausanne, Switzerland \\ ${ }^{5}$ Department of Radiology, Geneva University Hospital and Faculty of medicine, University of Geneva, \\ CH-1211 Genève 4, Switzerland \\ ${ }^{6}$ Paul Scherrer Institute, CH-5232 Villigen PSI, Switzerland \\ ${ }^{7}$ Department of Chemistry, Ecole Normale Supérieure, F-75230 Paris cedex 05, France
}

(Received 11 March 2010; published 1 July 2010)

\begin{abstract}
A high throughput method was designed to produce hyperpolarized gases by combining lowtemperature dynamic nuclear polarization with a sublimation procedure. It is illustrated by applications to ${ }^{129} \mathrm{Xe}$ nuclear magnetic resonance in xenon gas, leading to a signal enhancement of 3 to 4 orders of magnitude compared to the room-temperature thermal equilibrium signal at $7.05 \mathrm{~T}$.
\end{abstract}

DOI: 10.1103/PhysRevLett.105.018104

PACS numbers: 87.61. $-\mathrm{c}, 51.60 .+\mathrm{a}, 76.60 .-\mathrm{k}, 82.56 .-\mathrm{b}$

Hyperpolarization dramatically relieves nuclear magnetic resonance (NMR) from one of its main drawbacks, namely, the low sensitivity due to the weak nuclear spin polarization arising from the small energy splitting between nuclear spin levels. If the magnetic nuclei are hyperpolarized, one can make use of ca. $10^{-1}$ of the spins available in the sample, instead of probing merely ca. $10^{-5}$ of them. The impact of hyperpolarization in biomedical research is considerable, and new applications of major importance are expected in the coming years $[1,2]$.

Hyperpolarized ${ }^{129} \mathrm{Xe}$ has been extensively used for lung imaging, and its potential as blood tracer for in vivo vascular and perfusion imaging applications was demonstrated [3]. Following the introduction of the technique called HYPER-CEST [4], which exploits the fast exchange between xenon dissolved in a solvent and xenon trapped in cages attached to biosensors, new in vivo applications of hyperpolarized ${ }^{129} \mathrm{Xe}$ have been envisaged with potential uses in molecular imaging [5]. Hyperpolarized ${ }^{129} \mathrm{Xe}$ is also a powerful tool for studying surfaces, polymers, porosity of materials, and protein structures [6].

${ }^{129} \mathrm{Xe}$ and other isotopes of NMR-active noble gases can be polarized via optical pumping [7-10]. This method allows one to produce highly polarized gas, up to $70 \%$ for ${ }^{129} \mathrm{Xe}$ under optimum conditions, but large throughputs (1-2 L/h of xenon gas at atmospheric pressure) are only possible under less favorable conditions where the polarization level is limited (to about $20 \%$ in the case of ${ }^{129} \mathrm{Xe}$ ) [11].

It is also possible to greatly enhance the Boltzmann polarization by reducing the temperature to about $10 \mathrm{mK}$, and to increase the Zeeman energy by applying an external magnetic field on the order of $10 \mathrm{~T}[12,13]$. Compared to optical methods, the production rates could be increased by at least a factor 10 using this technique, but sophisticated cryogenics and large magnetic fields are required to obtain substantial polarization levels.

Dynamic nuclear polarization (DNP) combined with the so-called dissolution procedure yields ${ }^{13} \mathrm{C}$ nuclear polarization in liquids up to about $40 \%$ in selected molecules [14]. DNP is based on the transfer of polarization from the electron spins of paramagnetic centers embedded in a glassy frozen solution to neighboring nuclear spins [15]. For the technique to be efficient, the paramagnetic centers, usually stable radicals, have to be homogeneously distributed in the frozen solution containing the molecules of interest. The optimal temperature for an efficient polarization transfer is about $1 \mathrm{~K}$. The dissolution step rapidly transforms the frozen solution into a dilute roomtemperature solution in which the nuclear spins of the molecules of interest remain hyperpolarized. The technique is quite versatile and has been used to hyperpolarize ${ }^{6} \mathrm{Li},{ }^{13} \mathrm{C},{ }^{15} \mathrm{~N}$, and ${ }^{89} \mathrm{Y}$ in a variety of molecules in solution [16-19].

In this Letter, we propose to combine DNP with a sublimation procedure to hyperpolarize gases. This new method is used to hyperpolarize ${ }^{129} \mathrm{Xe}$ in xenon gas at natural isotope abundance.

Solid-state sample preparation.-Glassy matrices in which both the solidified gas and the paramagnetic centers (stable radicals) are homogeneously embedded were prepared. The triple point of xenon occurs at $161.4 \mathrm{~K}$ and $81.6 \mathrm{kPa}$ [20]. Because liquid xenon is nonpolar, a different solvent had to be used to dissolve nitroxide radicals such as TEMPO (2,2,6,6-Tetramethylpiperidine-1-oxyl) commonly used for DNP. We chose isobutanol (2-methylpropan-1-ol) because of its freezing point of $165 \mathrm{~K}$, which is only $3.6 \mathrm{~K}$ above the xenon triple point. At a pressure of 
$500 \mathrm{kPa}$, the temperature range in which both xenon and isobutanol are liquids is on the order of $30 \mathrm{~K}$. To prepare a $2 \mathrm{~mL}$ glassy frozen sample, the procedure was as follows: first, $1 \mathrm{~mL}$ isobutanol doped with $60 \mathrm{mM}$ TEMPO was incorporated into a homebuilt cold finger with a graduated glass. The cold finger was pressurized to $500 \mathrm{kPa}$ with xenon gas (natural isotopic abundance in the present study) and the bottom of the cold finger was slowly placed in a cell cooled by cold nitrogen gas.

Once the bottom of the cold finger had reached a temperature of $170 \pm 10 \mathrm{~K}$, liquid xenon formed on top of the isobutanol solution. By adjusting the temperature of the cold finger, the volume of liquid xenon could be controlled. The optimal ratio between liquid xenon and isobutanol was determined empirically through preliminary studies. In the present case, 1:1 liquid xenon:isobutanol solutions were prepared using the cold finger graduation, leading to a total volume of $2 \mathrm{~mL}$. After intermittently agitating the mixture for ca. $1 \mathrm{~min}$, a homogeneous solution containing $30 \mathrm{mM}$ of TEMPO was obtained. The solution was then frozen by plunging the cold finger in liquid nitrogen, and the glassy frozen sample was extracted from the cold finger before its transfer to the sample holder of the polarizer.

Dynamic nuclear polarization.-A volume of about $0.4 \mathrm{~mL}$ of the isobutanol-xenon solid mixture doped with TEMPO was inserted in the sample holder compatible with two custom-designed DNP polarizers [21]. DNP was performed at $1.2 \mathrm{~K}$ in either a $3.35 \mathrm{~T}$ or a $5 \mathrm{~T}$ polarizer. The hardware was previously described elsewhere and used for many dissolution DNP experiments $[21,22]$. The sample was irradiated at 93.94 or $139.85 \mathrm{GHz}$ with $30 \mathrm{~mW}$ microwave power (ELVA-1) for $15 \mathrm{~min}$. The time evolution of the ${ }^{129} \mathrm{Xe}$ polarization was monitored by NMR using a dedicated homebuilt spectrometer and a low-temperature NMR probe with remote tuning and matching [23].

Solid-state ${ }^{129} \mathrm{Xe}$ NMR measurements led to the determination of the nuclear polarization $P=5.1 \pm 0.4 \%$ at $3.35 \mathrm{~T}$ and $P=7.2 \pm 0.5 \%$ at $5 \mathrm{~T}$ (see Fig. 1 ), where $P$ is the ratio between the population difference of the two nuclear spin energy levels and the total number of nuclear spins. These values were calculated by multiplying the theoretical equilibrium Boltzmann polarization at $1.2 \mathrm{~K}$, $P_{\text {thermal }}$, by the ratio of the signal amplitudes measured after DNP and without DNP. The polarization increased following a single exponential behavior $P(t)=P_{\text {thermal }}+$ $P(t=\infty)\left[1-\exp \left(-t / \tau_{\mathrm{DNP}}\right)\right]$ with characteristic polarization time constants $\tau_{\mathrm{DNP}}=122 \pm 5 \mathrm{~s}$ at $3.35 \mathrm{~T}$ and $\tau_{\mathrm{DNP}}=141 \pm 7 \mathrm{~s}$ at $5 \mathrm{~T}$.

Sublimation and storage.-Once the ${ }^{129}$ Xe polarization reached its maximum value at low temperature, the sample was lifted above the helium bath and subsequently sublimated by injecting hot water vapor $(1.5 \mathrm{~mL}$ pressurized with helium gas to $0.6 \mathrm{MPa}$ at room temperature and heated to $440 \mathrm{~K}$ to reach a pressure of $1 \mathrm{MPa}$ ). A custom-designed sublimation insert compatible with the DNP polarizer was

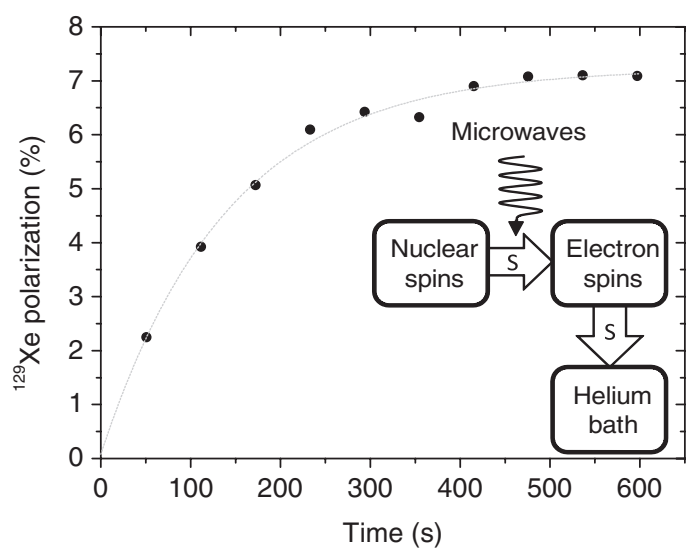

FIG. 1. ${ }^{129} \mathrm{Xe}$ polarization buildup measured at $1.2 \mathrm{~K}$ in $B_{0}=$ $5 \mathrm{~T}$. The gray dashed curve corresponds to the function $P(t)=$ $P_{\text {thermal }}+P(t=\infty)\left[1-\exp \left(-t / \tau_{\mathrm{DNP}}\right)\right]$ fitted to the data. The observed characteristic time constant is $\tau_{\mathrm{DNP}}=141 \pm 7 \mathrm{~s}$. A sketch representing the thermodynamical description of DNP is shown: the microwave irradiation pumps entropy $(S)$ from the nuclear spins to the electron spins from where it is dumped to the liquid helium bath. The overall result is a "cooling" of the nuclear spins, i.e., an increase of their polarization above the value expected from the bath temperature.

used. The insert is similar to the dissolution insert described in a previous publication [21], the main difference being that the output line has to be adapted to the gas output flow. A $3 \mathrm{~m}$ long, $2 \mathrm{~mm}$ inner diameter polytetrafluoroethylene output line was used in the present study. The extracted gas was separated from isobutanol and water in a homemade separator inserted along the output line. The resulting hyperpolarized xenon gas was collected and frozen in a cold finger immersed in liquid nitrogen $(77 \mathrm{~K})$ located in the center of a $0.1 \mathrm{~T}$ permanent magnet. A membrane vacuum pump was used to evacuate undesirable air and helium from the cold finger. The ${ }^{129} \mathrm{Xe}$ nuclear spin-lattice relaxation time under such storage conditions exceeds $10^{4} \mathrm{~s}$ [24], thus allowing for the transport of hyperpolarized xenon to distant sites and magnetic resonance instruments. Finally, xenon was extracted by a second sublimation step prior to performing the gas-phase measurements at room temperature. The procedure yielded $100 \mathrm{~mL}$ of hyperpolarized xenon gas at atmospheric pressure in less than 15 min (including sample loading, polarization, sublimation, and transfer) using less than $1 \mathrm{~L}$ of liquid helium. All 6 steps leading to the preparation of hyperpolarized ${ }^{129} \mathrm{Xe}$ are sketched in Fig. 2.

Hyperpolarized ${ }^{129} \mathrm{Xe}$ gas NMR.-The ${ }^{129} \mathrm{Xe}$ nuclear spin polarization of the resulting room-temperature hyperpolarized gas was measured in a high-resolution $7.05 \mathrm{~T}$ Avance NMR spectrometer (Bruker). We used a $5 \mathrm{~mm}$ broadband inverse probe (BBI probe, Bruker). The pressure in the NMR tube was stabilized at $5 \mathrm{kPa}$ above atmospheric pressure using an overpressure valve to maintain xenon in the tube and avoid exchange with air. The 


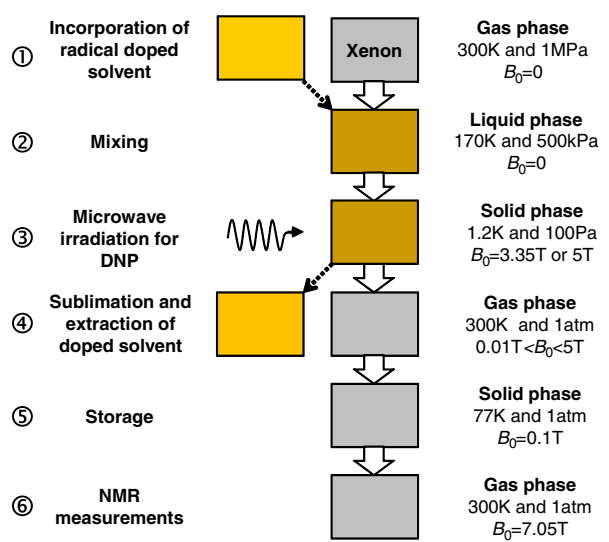

FIG. 2 (color online). Sketch representing the six-step procedure to hyperpolarize ${ }^{129} \mathrm{Xe}$ via DNP and sublimation. The temperature, the pressure, as well as the external magnetic field $B_{0}$ are specified for each step.

temperature was maintained at $25^{\circ} \mathrm{C}$ using a thermostated air flow. Adjustment of the shim coils as well as tuning and matching of the NMR probe were performed on a reference sample prior to the measurements of hyperpolarized xenon gas. Pulse length and amplitude were determined using a 1.1 MPa xenon gas sample sealed in a high-pressure NMR tube (Wilmad).

Figure 3 shows the NMR signal from a $5 \mathrm{~mm}$ diameter tube filled with hyperpolarized xenon at a pressure of $5 \mathrm{kPa}$ above atmospheric pressure acquired after a single $90^{\circ}$ pulse and the NMR signal from a fully relaxed xenon gas sample pressurized to $1.1 \mathrm{MPa}$ averaged over 128 experiments with $90^{\circ}$ pulses separated by relaxation intervals of $100 \mathrm{~s}$. The measured enhancement is 8200 , which amounts

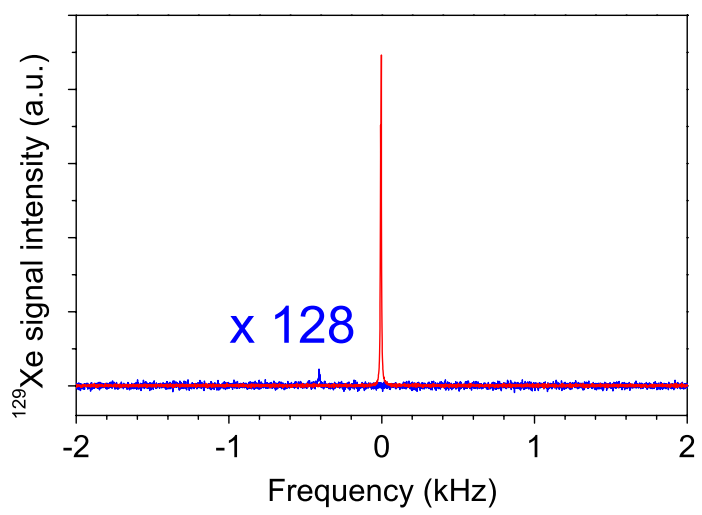

FIG. 3 (color online). Light gray (red): Fourier transform of a hyperpolarized ${ }^{129} \mathrm{Xe}$ free induction decay following a single $90^{\circ}$ pulse. The pressure of the hyperpolarized gas was $5 \mathrm{kPa}$ above atmospheric pressure. Dark gray (blue): Fourier transform (multiplied by a factor 128) of the free induction decay from a fully relaxed xenon gas sample pressurized to $1.1 \mathrm{MPa}$ averaged over 128 experiments with $90^{\circ}$ pulses separated by relaxation intervals of $100 \mathrm{~s}$. The acquisitions were performed at $7.05 \mathrm{~T}$ and $25^{\circ} \mathrm{C}$. to a nuclear spin polarization of $5.5 \%$. By comparing this value with polarization measurements in the solid state, we concluded that the polarization was negligibly altered by the sublimation and sample transfer procedure.

The sublimation step was only performed on samples polarized at $3.35 \mathrm{~T}$. From the $5 \mathrm{~T}$ solid-state polarization measurements, the polarization after sublimation is expected to be $7.2 \pm 0.5 \%$, corresponding to an enhancement of 10900 .

Discussions and conclusions. - It is essential to maintain at all times the hyperpolarized molecules or atoms in a sufficiently large magnetic field in order to minimize polarization losses due to spin-lattice relaxation. Spin-lattice relaxation times generally depend on magnetic field and temperature. In particular, they can become extremely short when the sample undergoes a phase transition. For instance, it was shown that the ${ }^{129} \mathrm{Xe}$ spin-lattice relaxation time in solid xenon is strongly dependent on the external magnetic field in the vicinity of the sublimation temperature [24]. It is therefore crucial to carry out the sublimation step inside the polarizer magnet bore as quickly as possible.

The present sublimation DNP technique is not restricted to noble gases, although for most polyatomic gases the polarization would not survive because of spin-rotation relaxation [25]. In such cases, it might be of interest to dissolve the solidified hyperpolarized gas directly in a viscous solvent.

It was recently shown that volumes as large as $13.5 \mathrm{~mL}$ can be hyperpolarized via DNP [26]. If half of this volume consists of solid xenon, the other half being composed of a frozen isobutanol solution containing TEMPO, such a setup would allow for the production of more than $3 \mathrm{~L}$ of hyperpolarized xenon at atmospheric pressure in about $15 \mathrm{~min}$, since the characteristic polarization time constant is on the order of $\tau_{\mathrm{DNP}}=140 \mathrm{~s}$. This is of major interest for potential clinical applications of hyperpolarized xenon in lung imaging or for a centralized production of hyperpolarized ${ }^{129} \mathrm{Xe}$ [27]. The main technical challenge that will need to be addressed for multiliter production of hyperpolarized gases will be the handling of the large volume of gas that will results from the sublimation process, although this problem is merely a technical problem that could be overcome by performing several simultaneous sublimations of samples located in compartmentalized volumes.

The optimization of the composition of the solid sample is expected to lead to further enhancements of the ${ }^{129} \mathrm{Xe}$ polarization. Replacing protons by deuterons was shown to double the maximum ${ }^{13} \mathrm{C}$ polarization in ${ }^{13} \mathrm{C}$-labeled acetate and glycine [28]. Using photoinduced radicals [29] created directly in liquid xenon would remove the need for an additional solvent and would double the throughput. Most importantly, using ${ }^{129} \mathrm{Xe}$-enriched xenon gas would shorten the polarization time constants through enhanced 
spin diffusion. Since cross relaxation to the fast relaxing quadrupolar ${ }^{131} \mathrm{Xe}$ spins is the most efficient spin-lattice relaxation mechanism of ${ }^{129} \mathrm{Xe}$ at low temperature [30], reducing the concentration of ${ }^{131} \mathrm{Xe}$ spins would most likely lead to an increase of the final ${ }^{129}$ Xe polarization.

In conclusion, we have demonstrated that large volumes of hyperpolarized gases can be rapidly obtained by combining low-temperature dynamic nuclear polarization with a sublimation procedure. A few challenges remain to be addressed for a multiliter production of highly polarized gases but, apart from the optimization of the solid-state composition required to obtain larger polarization, these are mainly technical issues that are expected to be readily overcome. The experimental setup designed to produce hyperpolarized gases is based on a DNP polarizer capable of hyperpolarizing a variety of nuclei whereas, to date, a dedicated apparatus was required for the production of highly polarized gases.

We are deeply grateful to Dr. Jacques van der Klink for initiating the DNP projects in Lausanne and for his invaluable technical help. This work was supported by the Swiss National Science Foundation (Grant No. 200020_124901 to A.C., "Ambizione" Grant No. PZOOP2_121928 to P. V., Grant No. 200020_124694 to G. B. and P. V.), the National Competence Center in Biomedical Imaging (NCCBI), the Centre d'Imagerie BioMédicale (CIBM) of the UNIL, UNIGE, HUG, CHUV, EPFL, and the Leenards and Jeantet Foundations.

*arnaud.comment@epfl.ch

[1] B. Driehuys, Science 314, 432 (2006).

[2] F. A. Gallagher, M. I. Kettunen, S. E. Day, D. E. Hu, J. H. Ardenkjaer-Larsen, R. in't Zandt, P. R. Jensen, M. Karlsson, K. Golman, M. H. Lerche, and K. M. Brindle, Nature (London) 453, 940 (2008).

[3] A. Cherubini and A. Bifone, Prog. Nucl. Magn. Reson. Spectrosc. 42, 1 (2003).

[4] L. Schroder, T. J. Lowery, C. Hilty, D. E. Wemmer, and A. Pines, Science 314, 446 (2006).

[5] O. Taratula and I. J. Dmochowski, Curr. Opin. Chem. Biol. 14, 97 (2010).

[6] K. Bartik, P. Choquet, A. Constantinesco, G. Duhamel, J. Fraissard, J.-N. Hyacinthe, J. Jokisaari, E. Locci, T. J. Lowery, M. Luhmer, T. Meersmann, I. L. Moudrakovski, G.E. Pavlovskaya, K.L. Pierce, A. Pines, J.A. Ripmeester, V.-V. Telkki, and W.S. Veeman, Actual. Chim. 287, 16 (2005).

[7] T. G. Walker and W. Happer, Rev. Mod. Phys. 69, 629 (1997).

[8] B. C. Grover, Phys. Rev. Lett. 40, 391 (1978).

[9] W. Happer, E. Miron, S. Schaefer, D. Schreiber, W. A. van Wijngaarden, and X. Zeng, Phys. Rev. A 29, 3092 (1984).
[10] A. Kastler, J. Phys. Radium 11, 255 (1950).

[11] I. C. Ruset, S. Ketel, and F. W. Hersman, Phys. Rev. Lett. 96, 053002 (2006).

[12] G. Frossati, J. Low Temp. Phys. 111, 521 (1998).

[13] E. V. Krjukov, J. D. O'Neill, and J. R. Owers-Bradley, J. Low Temp. Phys. 140, 397 (2005).

[14] J.H. Ardenkjaer-Larsen, B. Fridlund, A. Gram, G. Hansson, L. H. M. H. Lerche, R. Servin, M. Thaning, and K. Golman, Proc. Natl. Acad. Sci. U.S.A. 100, 10158 (2003).

[15] A. Abragam and M. Goldman, Nuclear Magnetism: Order and Disorder (Clarendon, Oxford, 1982).

[16] R. B. van Heeswijk, K. Uffmann, A. Comment, F. Kurdzesau, C. Perazzolo, C. Cudalbu, S. Jannin, J. Konter, P. Hautle, B. van den Brandt, G. Navon, J. van der Klink, and R. Gruetter, Magn. Reson. Med. 61, 1489 (2009).

[17] J. H. Ardenkjaer-Larsen, K. Golman, and K. M. Brindle, Molecular Imaging: Principles and Practice (McGrawHill, New York, 2010).

[18] C. Gabellieri, S. Reynolds, A. Lavie, G. S. Payne, M. O. Leach, and T. R. Eykyn, J. Am. Chem. Soc. 130, 4598 (2008).

[19] M. E. Merritt, C. Harrison, Z. Kovacs, P. Kshirsagar, C. R. Malloy, and A. D. Sherry, J. Am. Chem. Soc. 129, 12942 (2007).

[20] CRC Handbook of Chemistry and Physics, edited by David R. Lide (CRC Press/Taylor and Francis, Boca Raton, FL, 2009), 90th ed.

[21] A. Comment, B. van denBrandt, K. Uffmann, F. Kurdzesau, S. Jannin, J.A. Konter, P. Hautle, W. T. Wenckebach, R. Gruetter, and J. J. van der Klink, Concepts Magn. Reson. 31B, 255 (2007).

[22] S. Jannin, A. Comment, F. Kurdzesau, J. A. Konter, P. Hautle, B. van den Brandt, and J. J. van der Klink, J. Chem. Phys. 128, 241102 (2008).

[23] A. Comment, B. van den Brandt, K. Uffmann, F. Kurdzesau, S. Jannin, J. A. Konter, P. Hautle, W. T. Wenckebach, R. Gruetter, and J. J. van der Klink, Appl. Magn. Reson. 34, 313 (2008).

[24] N. N. Kuzma, B. Patton, K. Raman, and W. Happer, Phys. Rev. Lett. 88, 147602 (2002).

[25] R. G. Gordon, J. Chem. Phys. 44, 228 (1966).

[26] A. Comment, J. Rentsch, F. Kurdzesau, S. Jannin, K. Uffmann, R. van Heeswijk, P. Hautle, J. Konter, B. van den Brandt, and J. van der Klink, J. Magn. Reson. 194, 152 (2008).

[27] B. Driehuys, H. E. Moeller, Z. I. Cleveland, J. Pollaro, and L. W. Hedlund, Radiology 252, 386 (2009).

[28] F. Kurdzesau, B. van den Brandt, A. Comment, P. Hautle, S. Jannin, J. J. van der Klink, and J. A. Konter, J. Phys. D 41, 155506 (2008).

[29] M. D. Cook and B.P. Roberts, J. Chem. Soc. Chem. Commun. 1983, 264 (1983).

[30] S. Lang, I. L. Moudrakovski, C. I. Ratcliffe, J. A. Ripmeester, and G. Santyr, Appl. Phys. Lett. 80, 886 (2002). 